\title{
Syntactic Interchangeability in Word Embedding Models
}

\author{
Daniel Hershcovich \\ Assaf Toledo \\ Alon Halfon \\ IBM Research \\ daniel.hershcovich@gmail.com, \\ assaf.toledodibm.com, \\ \{alonhal, noams\}@il.ibm.com \\ Noam Slonim
}




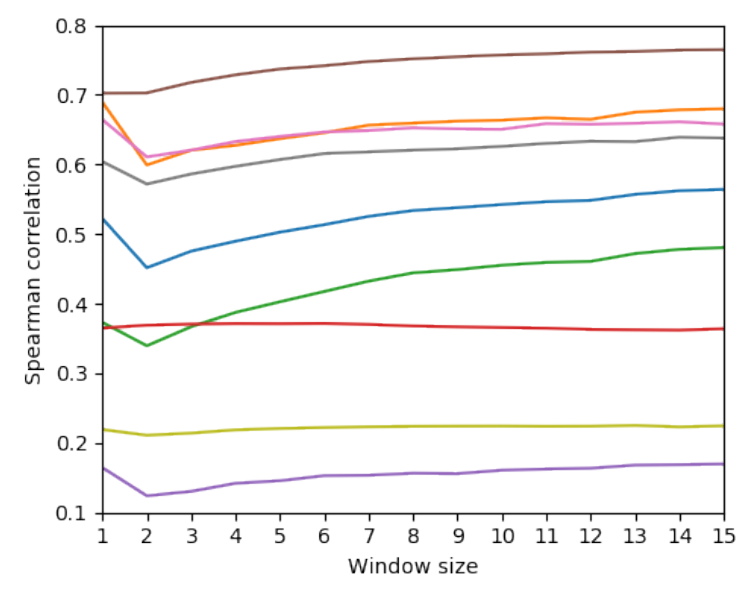

(a) CBOW

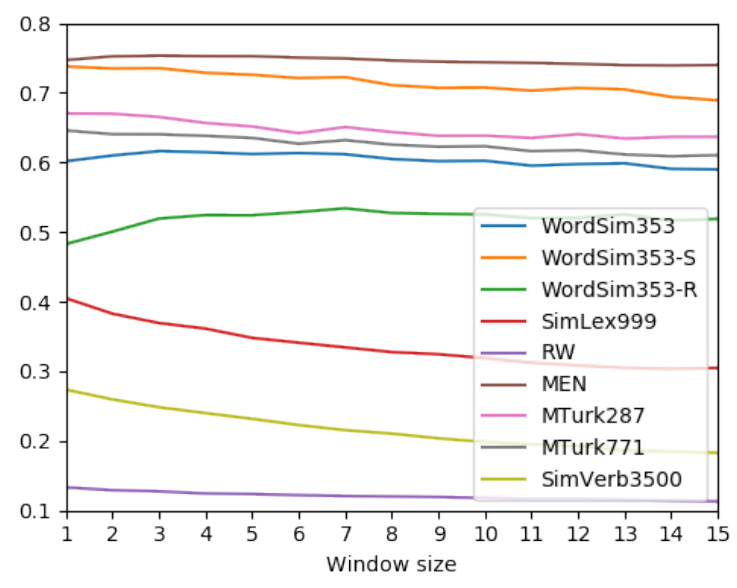

(b) SGNS

Figure 1: Performance of the CBOW (a) and SGNS (b) algorithms on each benchmark, for each window size, measured by Spearman correlation between the benchmark score and the word embedding cosine similarity.

categorize words based on the distributed representation encoded in word embeddings. We focus on similarity and relatedness, and evaluate word embedding models on several common benchmarks.

\subsection{Data}

We learn word embeddings from English Wikipedia, using a dump from May 1, 2017. ${ }^{2}$ The data is preprocessed using a publicly available preprocessing script, ${ }^{3}$ extracting text, removing nonalphanumeric characters, converting digits to text, and lowercasing the text.

Benchmarks. We use the following benchmarks: WordSim-353 (Finkelstein et al., 2001) and its partition into WordSim-353-Sim (Agirre et al., 2009) and WordSim-353-Rel (Zesch et al., 2008), SimLex999 (Hill et al., 2015), Rare Words (RW; Luong et al., 2013), MEN (Bruni et al., 2012), MTurk287 (Radinsky et al., 2011), MTurk-771 (Halawi et al., 2012), and SimVerb-3500 (Gerz et al., 2016). See Table 1 for the size of each benchmark.

\subsection{Hyper-parameters}

We use fastText (Bojanowski et al., 2017) to learn 300-dimensional word embedding models, using both the CBOW (continuous bag-of-words) and SGNS (skip-gram with negative sampling) algorithms (Mikolov et al., 2013). The context window size varies from 1 up to 15 . We include only all

\footnotetext{
${ }^{2}$ https://dumps.wikimedia.org/enwiki

${ }^{3}$ http: //mattmahoney. net/dc/textdata. html
}

words occurring 500 times or more (including function words), to avoid very rare words or uncommon spelling errors from skewing the results. All other hyper-parameters are set to their default values.

\subsection{Evaluation on Benchmarks}

To investigate the effect of window size on a model's performance on the benchmarks, we evaluate each model on each benchmark, using cosine similarity as the model's prediction for each pair. The performance is measured by Spearman correlation between the benchmark score and the word embedding cosine similarity (Levy et al., 2015).

Results. Figure 1 displays the performance of the CBOW and SGNS algorithms on each benchmark, with window sizes 1 to 15 . Apart from a small dip between windows 1 and 2 for CBOW, the performance is either nearly constant, or changes nearly monotonically with window size in each setting.

The relative improvement (or deterioration), in percents, with the increase of window size from 2 to 15 , are shown in Table 1 ( $\Delta$ win $=2 \rightarrow 15(\%)$ ). Interestingly, CBOW exhibits a positive correlation of window size with model's performance for all benchmarks but SimLex999, while performance for SGNS barely changes with window size, except for SimLex999 and SimVerb3500, where we see a strong negative correlation.

Discussion. In SimLex999 and in SimVerb3500, the words in each pair have the same part of speech by design (in particular, SimVerb3500 only contains verbs). Hypothesizing that the effect of win- 


\begin{tabular}{|c|c|c|c|c|c|c|c|c|}
\hline \multirow[b]{2}{*}{ Benchmark } & \multirow[b]{2}{*}{ Size } & \multicolumn{2}{|c|}{$\Delta$ win $=2 \rightarrow 15(\%)$} & \multicolumn{2}{|c|}{ \# Related } & \multicolumn{2}{|c|}{ \# Unrelated } & \multirow[b]{2}{*}{ p-value } \\
\hline & & CBOW & SGNS & All & Same-POS & All & Same-POS & \\
\hline WordSim353 & 353 & 24 & -3 & 122 & 107 & 53 & 40 & 0.038 \\
\hline WordSim353-S & 203 & 13 & -6 & 60 & 53 & 53 & 40 & 0.061 \\
\hline WordSim353-R & 252 & 42 & 4 & 104 & 89 & 39 & 31 & 0.26 \\
\hline SimLex999 & 999 & -1 & -20 & 234 & 199 & 334 & 295 & 0.897 \\
\hline RW & 2034 & 37 & -12 & 944 & 555 & 262 & 144 & 0.149 \\
\hline MEN & 3000 & 9 & -2 & 791 & 564 & 781 & 439 & $3 \cdot 10^{-10}$ \\
\hline MTurk287 & 287 & 8 & -5 & 49 & 39 & 119 & 68 & 0.004 \\
\hline MTurk771 & 771 & 12 & -5 & 204 & 153 & 200 & 146 & 0.365 \\
\hline SimVerb3500 & 3500 & 6 & -30 & 633 & 265 & 1217 & 566 & 0.974 \\
\hline
\end{tabular}

Table 1: Analysis of interchangeability (by same-POS) in word similarity and relatedness benchmarks. $\Delta$ win $=$ $2 \rightarrow 15(\%)$ is the relative change, in percents, of the model's performance (by Spearman correlation) when going from window size 2 to window size 15, for the CBOW and SGNS algorithms (\$2.3). Related and Unrelated are the top and bottom $30 \%$ of the pairs, by benchmark score, respectively. P-value is calculated using the hypergeometric test, comparing the enrichment of interchangeable pairs within related pairs, with a background of all related and unrelated pairs $(\S 3.1)$.

dow size is related to the model's implicitly learned concept of part of speech, we investigate this idea in the next section.

\section{Syntactic Interchangeability}

A word's part of speech (also known as syntactic category) is determined by syntactic distribution, and conveys information about how a word functions in the sentence (Carnie, 2002). We can generally substitute each word in a sentence with various words that are of the same part of speech, but not words that are of different parts of speech. While the same syntactic function can sometimes be fulfilled by words of various parts of speech or possibly longer phrases (such as adverbs and prepositional phrases, or multi-word expressions), part of speech is nonetheless a very good proxy for syntactic distribution (Mohammad and Pedersen, 2004).

Related to our work, Vulić et al. (2017) introduced a framework for automatic selection of specific context configurations for word embedding models per part of speech, improving performance on the SimLex999 benchmark. We take a different approach, investigating existing word embedding models and the way in which part of speech is reflected in them.

We define two words to be (syntactically) interchangeable if they share the same part of speech. We quantify interchangeability as a property of a word embedding model, as the proportion of words with the same part of speech within the list of nearest neighbors (that is, the most similar words according to the model) for each word in a pre-determined vocabulary. The higher the interchangeability ratio is, the more importance we assume the model implicitly places on interchangeability for the calculation of word similarity.

\subsection{Interchangeability Analysis in Word Similarity Benchmarks}

While all benchmarks we experiment with assign a score along a scale to each pair (calculated from human scoring), for our experiment we would like to use a binary annotation of whether a pair is related or not. For this purpose, we divide the whole range of scores, for each benchmark, to three parts: the lowest $30 \%$ of the range between the lowest and highest scores is considered "unrelated", the top $30 \%$ as "related", and the middle $40 \%$ are ignored. 
Interchangeability enrichment. Given the binary classification obtained from the humanannotated scores for each benchmark, we can find the enrichment of interchangeable pairs among related pairs. We use spaCy $2.0 .11^{4}$ (with the en_core_web_sm model) to annotate the POS for each word in each benchmark pair (tagging them in isolation to select the most probable POS), and look at the set of same-POS pairs in the benchmark. For each of the benchmarks, we calculate a p-value using the hypergeometric test, comparing the enrichment of same-POS pairs within related pairs, with a background distribution of all related and unrelated pairs (ignoring ones in the middle $40 \%$ range of scores).

Results. Table 1 shows the enrichment of interchangeable pairs among related and unrelated pairs for each benchmark. For WordSim353, MEN and MTurk287, the set of related pairs contains significantly more interchangeable pairs than the background set $(p<0.05),{ }^{5}$ suggesting that these benchmarks are particularly sensitive to POS.

\subsection{Nearest Neighbor Analysis}

To try and relate the results from $\S 2.3$ and $\$ 3.1$, we measure the relation between window size and interchangeability by analyzing nearest neighbors in word embedding models. In our experiment, the nearest neighbors of a word are the words with the highest cosine similarity between their vectors.

Collecting pivots. We create a word list for each of the three most common parts of speech: nouns, adjectives and verbs. For each POS, we list all lemmas of all synsets of that POS from WordNet (Miller, 1998). To "purify" the lists and avoid noise from homonyms, we remove from each list any lemma that also belongs to a synset from another POS. As a further cleaning step, we use spaCy to tag each word, and only keep words for which the spaCy POS agrees with the WordNet POS. Without context, spaCy will likely choose the most common POS based on its training corpus, which is different from WordNet, increasing the robustness.

This process results in 6407 uniquely-noun, 2784 uniquely-adjective and 1460 uniquely-verb words, which we refer to as our pivot lists.

\footnotetext{
${ }^{4}$ https://spacy.io

${ }^{5}$ The fact that not all pairs in SimLex 999 and SimVerb3500 are judged as interchangeable in our experiment is due to ambiguity: for some words, spaCy selected a POS which is not the one intended when constructing the benchmark.
}

\begin{tabular}{l|ccc|ccc|ccc} 
Algo- & \multicolumn{3}{|c|}{ NOUN } & \multicolumn{3}{|c|}{ ADJ } & \multicolumn{3}{|c}{ VERB } \\
rithm & 1 & 15 & $\mathrm{r}$ & 1 & 15 & $\mathrm{r}$ & 1 & 15 & $\mathrm{r}$ \\
\hline CBOW & 79 & 70 & -0.96 & 72 & 48 & -0.93 & 55 & 41 & -0.91 \\
SGNS & 78 & 66 & -0.95 & 66 & 39 & -0.94 & 51 & 41 & -0.92
\end{tabular}

Table 2: Percentage of interchangeable neighbors per pivot POS for the smallest (1) and largest (15) windows in our experiment, for the CBOW and SGNS algorithms. The number of interchangeable neighbors has a strong negative Pearson correlation (r) with window size for windows 1 to 15 ( $p<0.01$, two-tailed t-test).

Calculating nearest neighbor POS. We find the 100 nearest neighbors for each word in our pivot lists, according to each fastText model with windows 1 through 15 . We filter these neighbors to keep only words in the spaCy vocabulary, and inspect the remaining top 10. Again using spaCy, we tag the POS of each neighbor in the result. We subsequently calculate a histogram, for each POS $x$, of its neighbor-POS $y$, that is, the POS assigned to the neighbors of words with POS $x$.

Results. Table 2 shows the results of this experiment. For nouns, adjectives and verbs, we consistently see a decrease in the number of same-POS neighbors when we increase the window size, relative to the total number of nearest neighbors.

Figure 2 shows the the absolute number of neighbors per algorithm, pivot POS and neighbor POS, for all window sizes we experimented with. The number of nearest neighbors of the same POS is consistently decreasing with window size, while the number of nearest neighbors of other POS are increasing or unaffected.

Discussion. The results clearly suggest that for both CBOW and SGNS, models with a larger window size are less likely to consider words of the same POS as strongly related. That is, syntactic interchangeability is negatively correlated with window size. This is in sharp contrast to our results from $\$ 2.3$, where performance for CBOW on almost all benchmarks (among them WordSim353, MEN and MTurk287, for which we showed that syntactic interchangeability plays a role) consistently improved with window size. We also find the conclusion to contradict the impression regarding SGNS, where SimLex999 and SimVerb3500 showed worse performance for larger windows: if 

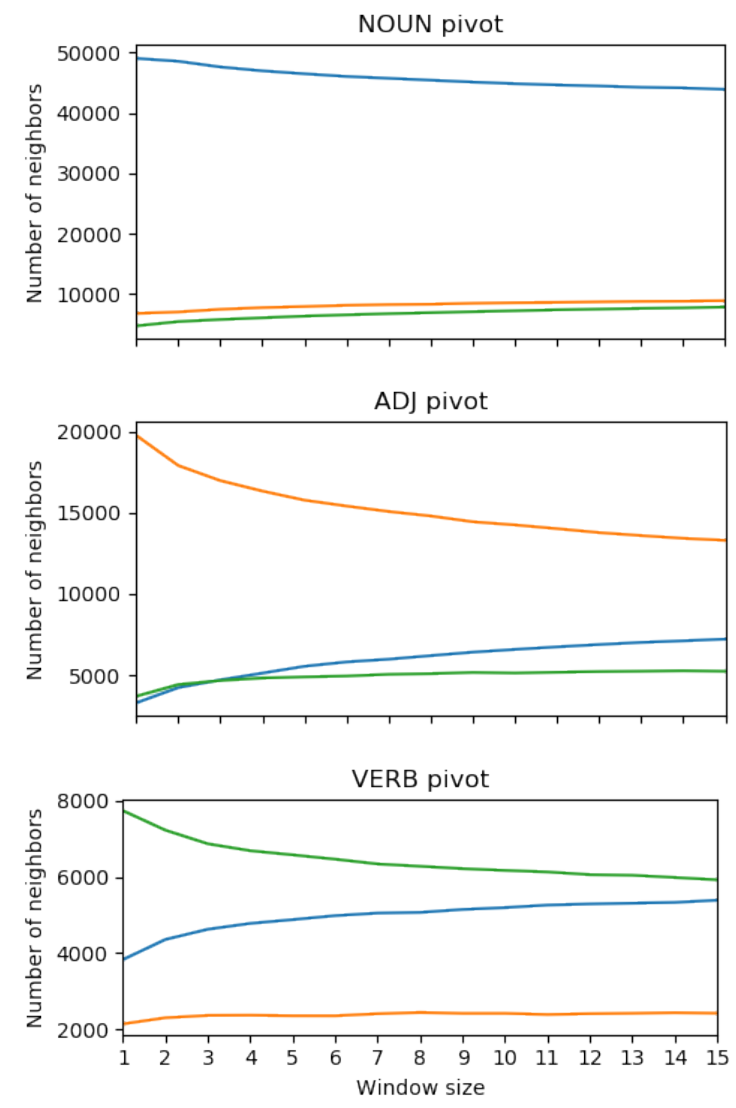

(a) CBOW
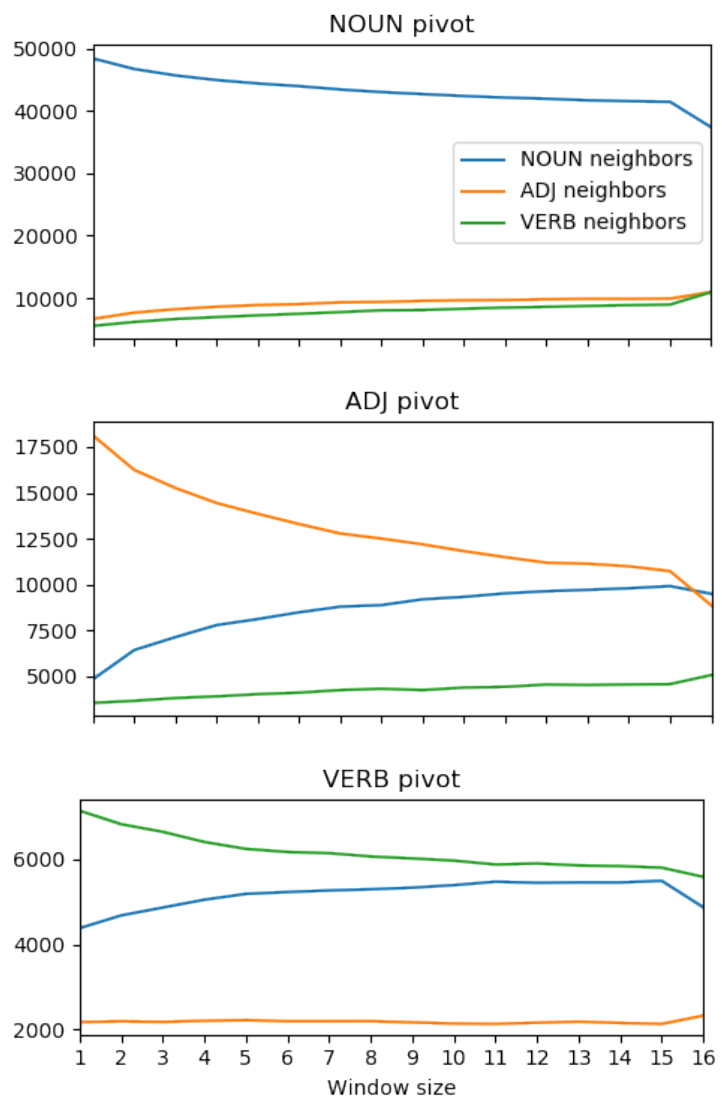

(b) SGNS

Figure 2: Number of neighbor per POS for each pivot POS and for each window size, for the CBOW (a) and SGNS (b) algorithms. The number of same-POS neighbors is consistently decreasing with window size.

POS should not play a role in these benchmarks, then models with a bias toward syntactic interchangeability (i.e., models with lower windows) should perform worse on these benchmarks.

\section{Conclusion}

We investigated the effect of the context window size hyper-parameter on the performance on word similarity benchmarks. We showed that (1) increasing the window size results in a lower probability of interchangeable nearest neighbors for both CBOW and SGNS algorithms; (2) in some widely used benchmarks, syntactic interchangeability increases the probability of similarity or relatedness; (3) increasing the window size typically improves performance in predicting similarity or relatedness for CBOW, but has little impact on SGNS.

SimLex 999 and SimVerb3500 proved to be exceptions to both (2) and (3), since all pairs in them are interchangeable by construction, but on them, increasing the window size has no effect for CBOW and negative impact for SGNS.
This contradiction is presented as a challenge to the community, and could perhaps be explained by other factors affected by window size.

Our investigation focused on a specific relation between words, namely whether they share a part of speech. Many other relations are of interest to the NLP community, such as syntactic dependency relations, and semantic relations like hypernymy and synonymy. Furthermore, a similar analysis could be applied to other word embedding hyperparameters, such as the vector dimension. While we used a constant vector dimension of 300 in our experiments, it is an open question whether models with different vector dimensions differ with respect to their tendency to capture different word relations. Future work will extend our analysis to other relations and hyper-parameters.

\section{Acknowledgments}

We thank the anonymous reviewers for their helpful comments. 


\section{References}

Eneko Agirre, Enrique Alfonseca, Keith Hall, Jana Kravalova, Marius Paşca, and Aitor Soroa. 2009. A study on similarity and relatedness using distributional and wordnet-based approaches. In Proceedings of Human Language Technologies: The 2009 Annual Conference of the North American Chapter of the Association for Computational Linguistics, pages 19-27. Association for Computational Linguistics.

Faisal Alshargi, Saeedeh Shekarpour, Tommaso Soru, Amit Sheth, and Uwe Quasthoff. 2018. Concept2vec: Metrics for evaluating quality of embeddings for ontological concepts. arXiv preprint arXiv:1803.04488.

Oded Avraham and Yoav Goldberg. 2016. Improving reliability of word similarity evaluation by redesigning annotation task and performance measure. arXiv preprint arXiv:1611.03641.

Amir Bakarov. 2018. A survey of word embeddings evaluation methods. arXiv preprint arXiv:1801.09536.

Mohit Bansal, Kevin Gimpel, and Karen Livescu. 2014. Tailoring continuous word representations for dependency parsing. In Proceedings of the 52nd Annual Meeting of the Association for Computational Linguistics (Volume 2: Short Papers), volume 2, pages 809-815.

Piotr Bojanowski, Edouard Grave, Armand Joulin, and Tomas Mikolov. 2017. Enriching word vectors with subword information. Transactions of the Association for Computational Linguistics, 5:135-146.

Elia Bruni, Gemma Boleda, Marco Baroni, and NamKhanh Tran. 2012. Distributional semantics in technicolor. In Proceedings of the 50th Annual Meeting of the Association for Computational Linguistics: Long Papers-Volume 1, pages 136-145. Association for Computational Linguistics.

Andrew Carnie. 2002. Syntax: A Generative Introduction (Introducing Linguistics). Blackwell Publishing.

Billy Chiu, Anna Korhonen, and Sampo Pyysalo. 2016. Intrinsic evaluation of word vectors fails to predict extrinsic performance. In Proceedings of the $1 s t$ Workshop on Evaluating Vector-Space Representations for NLP, pages 1-6.

Lev Finkelstein, Evgeniy Gabrilovich, Yossi Matias, Ehud Rivlin, Zach Solan, Gadi Wolfman, and Eytan Ruppin. 2001. Placing search in context: The concept revisited. In Proceedings of the 10th international conference on World Wide Web, pages 406414. ACM.

Daniela Gerz, Ivan Vulić, Felix Hill, Roi Reichart, and Anna Korhonen. 2016. SimVerb-3500: A LargeScale Evaluation Set of Verb Similarity. In EMNLP.
Yoav Goldberg. 2016. A primer on neural network models for natural language processing.

Guy Halawi, Gideon Dror, Evgeniy Gabrilovich, and Yehuda Koren. 2012. Large-scale learning of word relatedness with constraints. In Proceedings of the 18th ACM SIGKDD international conference on Knowledge discovery and data mining, pages 14061414. ACM.

Zellig S Harris. 1954. Distributional structure. Word, 10(2-3):146-162.

Felix Hill, Roi Reichart, and Anna Korhonen. 2015. Simlex-999: Evaluating semantic models with (genuine) similarity estimation. Computational Linguistics, 41(4):665-695.

Stanisław Jastrzebski, Damian Leśniak, and Wojciech Marian Czarnecki. 2017. How to evaluate word embeddings? on importance of data efficiency and simple supervised tasks. arXiv preprint arXiv:1702.02170.

Omer Levy, Yoav Goldberg, and Ido Dagan. 2015. Improving distributional similarity with lessons learned from word embeddings. Transactions of the Association for Computational Linguistics, 3:211-225.

Chu-Cheng Lin, Waleed Ammar, Chris Dyer, and Lori Levin. 2015. Unsupervised pos induction with word embeddings. arXiv preprint arXiv:1503.06760.

Pierre Lison and Andrey Kutuzov. 2017. Redefining context windows for word embedding models: An experimental study. In Proceedings of the 21st Nordic Conference on Computational Linguistics, pages 284-288. Association for Computational Linguistics.

Thang Luong, Richard Socher, and Christopher Manning. 2013. Better word representations with recursive neural networks for morphology. In Proceedings of the Seventeenth Conference on Computational Natural Language Learning, pages 104-113.

Tomas Mikolov, Kai Chen, Greg Corrado, and Jeffrey Dean. 2013. Efficient estimation of word representations in vector space. arXiv preprint arXiv:1301.3781.

George Miller. 1998. WordNet: An electronic lexical database. MIT press.

Saif Mohammad and Ted Pedersen. 2004. Combining lexical and syntactic features for supervised word sense disambiguation. In Proceedings of the Eighth Conference on Computational Natural Language Learning (CoNLL-2004) at HLT-NAACL 2004.

Jeffrey Pennington, Richard Socher, and Christopher Manning. 2014. GloVe: Global vectors for word representation. In Proceedings of the 2014 conference on empirical methods in natural language processing (EMNLP), pages 1532-1543. 
Kira Radinsky, Eugene Agichtein, Evgeniy Gabrilovich, and Shaul Markovitch. 2011. A word at a time: computing word relatedness using temporal semantic analysis. In Proceedings of the 20th international conference on World wide web, pages 337-346. ACM.

Tobias Schnabel, Igor Labutov, David Mimno, and Thorsten Joachims. 2015. Evaluation methods for unsupervised word embeddings. In Proceedings of the 2015 Conference on Empirical Methods in Natural Language Processing, pages 298-307.

Ivan Vulić, Roy Schwartz, Ari Rappoport, Roi Reichart, and Anna Korhonen. 2017. Automatic selection of context configurations for improved class-specific word representations. In Proceedings of the 21st Conference on Computational Natural Language Learning (CoNLL 2017), pages 112-122, Vancouver, Canada. Association for Computational Linguistics.

Dongqiang Yang and David Martin Powers. 2006. Verb similarity on the taxonomy of wordnet. In Proceedings of $G W C$.

Torsten Zesch, Christof Müller, and Iryna Gurevych. 2008. Using wiktionary for computing semantic relatedness. In $A A A I$, volume 8, pages 861-866. 